\title{
Rapid multi-locus sequence typing direct from uncorrected long reads using Krocus
}

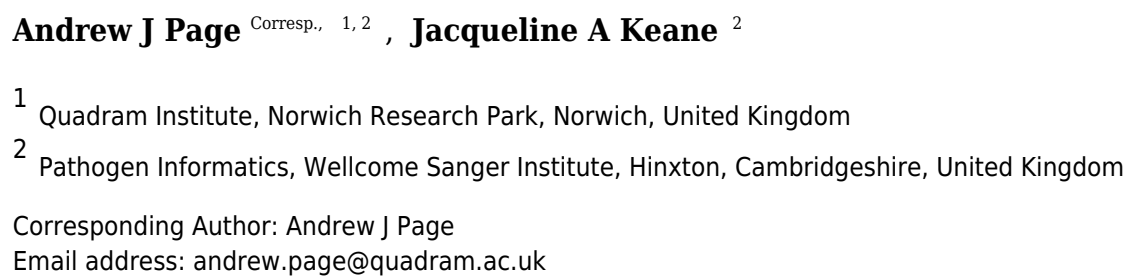

Genome sequencing is rapidly being adopted in reference labs and hospitals for bacterial outbreak investigation and diagnostics where time is critical. Seven gene multi-locus sequence typing is a standard tool for broadly classifying samples into sequence types, allowing, in many cases, to rule a sample out of an outbreak, or allowing for general characteristics about a bacterial strain to be inferred. Long read sequencing technologies, such as from Oxford Nanopore, can produce read data within minutes of an experiment starting, unlike short read sequencing technologies which require many hours/days. However, the error rates of raw uncorrected long read data are very high. We present Krocus which can predict a sequence type directly from uncorrected long reads, and which was designed to consume read data as it is produced, providing results in minutes. It is the only tool which can do this from uncorrected long reads. We tested Krocus on over 700 isolates sequenced using long read sequencing technologies from PacBio and Oxford Nanopore. It provides sequence types for isolates on average within 90 seconds, with a sensitivity of $94 \%$ and specificity of $97 \%$ on real sample data, directly from uncorrected raw sequence reads. The software is written in Python and is available under the open source license GNU GPL version 3. 
1 Rapid multi-locus sequence typing direct

2 from uncorrected long reads using

3 Krocus

4 Andrew J. Page ${ }^{1,2, *}$, Jacqueline A. Keane ${ }^{2}$

$5 \quad{ }^{1}$ Quadram Institute Bioscience, Norwich Research Park, Norwich, UK, NR4 7UA.

$6 \quad{ }^{2}$ Pathogen Informatics, Wellcome Sanger Institute, Wellcome Genome Campus, Cambs, UK, CB10 1 SA.

7 Corresponding email: andrew.page@quadram.ac.uk 


\section{Abstract:}

9 Genome sequencing is rapidly being adopted in reference labs and hospitals for bacterial

10 outbreak investigation and diagnostics where time is critical. Seven gene multi-locus sequence

11 typing is a standard tool for broadly classifying samples into sequence types, allowing, in many

12 cases, to rule a sample out of an outbreak, or allowing for general characteristics about a

13 bacterial strain to be inferred. Long read sequencing technologies, such as from Oxford

14 Nanopore, can produce read data within minutes of an experiment starting, unlike short read

15 sequencing technologies which require many hours/days. However, the error rates of raw

16 uncorrected long read data are very high. We present Krocus which can predict a sequence type

17 directly from uncorrected long reads, and which was designed to consume read data as it is

18 produced, providing results in minutes. It is the only tool which can do this from uncorrected long

19 reads. We tested Krocus on over 700 isolates sequenced using long read sequencing

20 technologies from PacBio and Oxford Nanopore. It provides sequence types for isolates on

21 average within 90 seconds, with a sensitivity of $94 \%$ and specificity of $97 \%$ on real sample data,

22 directly from uncorrected raw sequence reads. The software is written in Python and is available

23 under the open source license GNU GPL version 3.

\section{Introduction}

With rapidly falling costs, long read sequencing technologies, such as from Pacific Biosciences (PacBio) and Oxford Nanopore Technologies (ONT), are beginning to be used for outbreak investigations (Faria et al. 2017; Quick et al. 2015) and for rapid clinical diagnostics (Votintseva et al. 2017). Long read sequencers from Oxford Nanopore can produce sequence reads in a matter of minutes and sequencers from PacBio can produce sequences in a number of hours compared to short read sequencing technologies which takes hours/days. Seven gene multilocus sequence typing (MLST) is a widely used classification system for categorising bacteria. It can be used to quickly rule an isolate out of an outbreak and knowing a sequence type (ST) can often allow for general characteristics of a bacteria to be inferred. By reducing the time from swab to an actionable answer, genomics can begin to directly influence clinical decisions, with the potential to make a real positive impact for patients (Gardy and Loman 2018).

With the increased speed afforded by long read sequencing technologies comes increased base errors rates. The high error rates inherent in long read sequencing reads require specialised tools to correct the reads (Koren et al. 2017), however these methods have substantial computational resource requirements often taking longer to run than the original time to generate the sequencing data.

A full overview of MLST software for short read sequencing technologies is available in (Page et al. 2017). Of the software reviewed in (Page et al. 2017) only the methods which take a de novo assembly as input can be used with long read sequencing technologies, however de novo assembly has a substantial post processing computational overhead, which can exceed the time taken to perform the sequencing. StringMLST (Gupta, Jordan, and Rishishwar 2017), was designed to rapidly predict MLST from raw read sets by performing a $k$-mer analysis. MentaLiST 
49

50

51

52

53

54

55

56

57

58

59

60

61

62

63

64

\section{Materials and Methods}

66

67

68

69

70

71

72

73

74

75 MLST from uncorrected long read sequencing data. https://github.com/andrewjpage/krocus. allele file.

(Feijao et al. 2018) takes a similar k-mer analysis approach and is designed for large typing schemes, such as cgMLST and wgMLST. They were designed to work only with high base quality short read sequencing data. To our knowledge no method currently exists for calling

We present Krocus which can rapidly estimate sequence types directly from uncorrected long reads from isolates. Results are presented using the largest public dataset of bacterial long read data containing over 700 samples generated using the PacBio sequencing technology, and for real and simulated datasets of ONT data. On average it produces sequence correct sequence types in 90 seconds, with a sensitivity of $94 \%$ and specificity of $97 \%$ for uncorrected PacBio sequence data. On a dataset consisting of 524 simulated ONT samples based on E. coli reference genomes the sensitivity was $82-94 \%$ depending on the flowcell modelled. On a small real ONT dataset of $12 \mathrm{~K}$. pneumoniae a sensitivity of $100 \%$ was achieved. Krocus is the only tool which can call MLST directly from uncorrected long reads with high accuracy. It is written completely in Python 3 and is available under the open source licence GNU GPL 3 from

The basic method of Krocus is to take short $k$-mers and calculate the coverage over the MLST alleles and a flowchart showing the method is presented in Figure 1. As the base errors are mostly uniformly distributed, a well chosen $k$-mer value results in short stretches of error free bases. Some $k$-mers will be erroneously flagged due to errors however as more reads are added (above 5X), these errors are filtered out as they have a low occurrence overall.

Krocus takes as input the path to an MLST scheme, a FASTQ file containing uncorrected long reads from an isolate and a $k$-mer size. The MLST alleles are contained in 7 FASTA files, downloaded from PubMLST (Jolley and Maiden 2010) or taken from the set distributed with the software. Each sequence in the allele files contains a unique identifier and the combination of these allele identifiers gives rise to the sequence type (ST), contained within a profile tab delimited file. An alignment-free $k$-mer sequence analysis approach is used to determine the presence and absence of particular alleles, with optimisations for high error rate long read sequencing data. For a given $k$-mer size, the $k$-mers are extracted from each sequence in each

In long read sequencing reads, whilst there are high base error rates, the errors are mostly uniformly distributed. The ideal $k$-mer size is the mean number of bases on a read which is free from errors, for example if the base error rate $n$ is $91 \%$ an error occurs on average every $\sim 11$ bases, thus the $k$-mer size $k$ calculated as $k=\lfloor 100 /(100-n)\rfloor$. A $k$-mer size which is too high would invariably always include an erroneous base, reducing the probability of a match with the allele files. A $k$-mer size which is too low would reduce the possible $k$-mer space and lead to an increase in matches by random chance. Each long read is inspected and $k$-mers are generated with a step size of $k$ giving an average depth of $k$-mer coverage of 1 . If a single $k$-mer from this set is present in the allele $k$-mers, the read is kept for further analysis, if no $k$-mers are present, the read is discarded as it is unlikely to contain the MLST genes. 
93

94

95

96

97

98

99

100

101

102

103

104

105

106

107

108

109

110

All possible $k$-mers are generated for the read which passed the initial filtering with a step size of 1 , giving an average depth of $k$-mer coverage equal to $k$, with $k$-mers occuring more than 5 times excluded from further comparison as they do not impart useful information. For each allele file, the intersection of the allele $k$-mers and the read $k$-mers is taken. The read is split up into bins of length $\mathrm{k}$, and the intersecting $k$-mers are assigned to their corresponding bin in the read to produce an approximate $k$-mer coverage of the read. A sliding window (default 4 times $k$-mer size) is used to span short gaps, which are likely the result of small errors in the underlying sequencing data. The largest contiguous block of $k$-mer coverage in the read is identified, based on the sliding window results, and if it meets the minimum block size (default 150 bases, derived from $1 / 3$ of the average length 467 of all sequences in pubMLST, retrieved 02-02-18), it is said to contain one of the typing alleles. The block is extended by 100 bases on either side to ensure the full allele is captured. The $k$-mers matching this block in the read are extracted and $k$-mer counts corresponding to the allele matching $k$-mers are incremented. The read is reverse complemented and the same search is undertaken once more.

At defined intervals (default 200 reads) the genes of each allele are analysed to calculate the number of $k$-mers covered by the raw read, allowing for the input files to be streamed in realtime as data is generated. Only ONT sequencers support real-time streaming of reads. PacBio sequencers require post analysis once the sequencing experiment is complete. If an allele has a gene with $100 \% k$-mer coverage, it is said to be present, if it is less than $100 \%$, the allele with the most number of $k$-mers is identified, but with a low confidence flag. Where 2 or more alleles of the same gene have $100 \%$ coverage, the sequence with the highest $k$-mer coverage is used. Novel combinations of alleles and new, unseen, alleles cannot be reliably detected using this method, and so are excluded from the analysis.

\section{PacBio samples}

The NCTC 3000 project (http://www.sanger.ac.uk/resources/downloads/bacteria/nctc ) aims to produce 3000 bacteria reference sequences using the PacBio long read sequencing technology. Each of the reference strains was selected for sequencing to maximise diversity and to capture historically medically important strains. This is currently the largest public long read sequencing project for bacteria and is still on-going with 1048 assemblies publicly available (accessed $2 / 1 / 18$ ). The assemblies were downloaded from the project website and the sequencing reads directly from the European Nucleotide Archive. The sequencing reads were all generated on the PacBio RSII between 2014 and 2017. The assemblies used for comparison with Krocus were generated using an open source pipeline ( https://github.com/sanger-pathogens/vr-codebase ) which first performed a de novo assembly using HGAP (SMRT analysis version 2.3.0) (Chin et al. 2013), followed by circularisation with circlator (version 1.5.3) (Hunt et al. 2015), and finally automated polishing with the resequencing protocol (SMRT analysis version 2.3.0) from PacBio. Each assembly (1048) was sequence typed using the TS-m/st software (https://github.com/tseemann/mlst). Any samples identified as ambiguous or untypable by TS$\mathrm{m} / \mathrm{st}$ were excluded from further analysis. Also, samples where there were no corresponding MLST scheme for the species (339) were also excluded, as a meaningful comparison cannot be made. The TS-m/st software was shown in (Page et al. 2017) to never make any false positive ST calls. The remaining 709 samples are detailed in Supplementary Table 1, including accession numbers, and summarized in Table 1, covering 43 species and 638 STs with representatives 
137 from both gram positive and gram negative. The FASTQ files of the uncorrected reads were 138 generated from the raw data using the PacBio SMRTlink pipeline (version 5.0.1.9585), and the 139 time for this conversion is not considered in the results presented in this paper as it is a standard 140 preprocessing step required for many downstream analyses.

\section{PacBio control samples}

142 A set of 74 samples representing 48 species were selected as controls from the NCTC 3000 143 project. Each were sequenced using the PacBio long read sequencing technology as described 144 previously and are listed in Supplementary Table 2. The controls were selected from within the 145 same genus as the cases as listed in Table 1, but from different species. The species 146 classifications came from experimental techniques. The de novo assemblies of each sample 147 were analysed with the TS-m/st software, and any which resulted in valid sequence types were 148 removed to reduce the impact of confounders from misclassified isolates.

149

150

151

152

153

154

155

156

157

158

159

160

161

162

163

164

165

166

167

168

169

170

171

172

173

174

175

176

177

178

\section{ONT samples}

To analyse the performance of Krocus on ONT data we selected a set of $12 \mathrm{~K}$. pneumoniae samples used previously for performance comparisons in (R. Wick, Judd, and Holt 2018) and (R. R. Wick et al. 2017). The dataset is available from (R. Wick 2017a) and detailed in Supplementary Table 3. For comparison Canu (Koren et al. 2017) (version 1.5) assemblies and Unicycler (Wick et al. 2016) (version 0.4.0) assemblies, utilising Miniasm (Li 2016) and Racon (Vaser et al. 2017), post Nanopolish (https://github.com/jts/nanopolish) (version 0.7.0), created using only the long read data (R. Wick 2017b) were used.

There are currently no large publicly available ONT datasets. To overcome this, simulated ONT reads were generated using NanoSim-H (https://github.com/karel-brinda/NanoSim-H) (version 1.1.0.2), a derivative of NanoSim (Yang et al. 2017). NanoSim-H includes error models for simulating $E$. coli uncorrected nanopore reads with multiple flowcell types. Every complete $E$. coli reference genome in RefSeq was downloaded $(n=549)$. TS-mlst was run on these reference genomes to generate the target ST. TS-m/st was unable to call an E.coli ST in 25 cases and these were excluded from further analysis. Of the samples, 11 contained 1 or more incomplete gene, 3 contained duplicated genes, 2 were missing a gene, and one was a novel combination of alleles. Extended details of the samples and the results are listed in Supplementary Table 4. Using NanoSim-H 20,000 reads were simulated with the R9 flowcell error model (R9_1D and R9_2D) and the default parameters for each reference genome ( $n=524)$. The reads were converted from FASTA format to FASTQ format using PyFASTAQ (https://github.com/sangerpathogens/Fastaq) (version 3.17.0) and provided to Krocus (default parameters).

\section{Compute resources}

All experiments on real datasets were performed using the Wellcome Sanger Institute compute infrastructure running Ubuntu 12.04 LTS, with each host containing 32 cores (AMD Opteron Processor 6272) and 256GB of RAM. All experiments involving simulated datasets were performed using the MRC CLIMB (Connor et al. 2016) cloud infrastructure running the Genomics Virtual Lab (Afgan et al. 2015), with each host containing 8 cores and 64 GB of RAM. Only a single core was used in each performance experiment with the mean memory requirement of $0.354 \mathrm{~GB}$ (std dev 0.16 ). 


\section{Results}

\section{Pacbio Results}

182 Each of the assemblies from the NCTC dataset were run through TS-m/st to generate a ST. 183 Krocus was run for each sample using the uncorrected FASTQ files and default settings and 184 halted when the ST matched the expected result from TS-m/st. The time to achieve the correct 185 predicted ST was noted, as were the number of reads, with a mean of 86 seconds, after 186 processing a mean of 17,439 reads. The number of reads required before Krocus correctly predicts the ST is presented in Figure 2a, with only species with 10 or more isolates included. The running time for each species is presented in Figure $2 \mathrm{~b}$. The running time of $3 \mathrm{~S}$. aureus samples was elevated due to the need to process a higher than average number of reads, however within 60 seconds 6 out of 7 alleles had been correctly identified, with the last allele taking up to a further 11 minutes to identify correctly. In $94 \%$ of cases (sensitivity) the results from Krocus and TS-m/st were in agreement, with the calculations listed in Supplementary Table 5.

In 43 cases $(6 \%)$ STs did not match the expected ST or were untypable, with 40 of these calling 6 out of 7 typing genes correctly. In the remaining 1 case, 5 out of 7 genes were called correctly. These failures are due to known systematic errors with long homoployers with the PacBio sequencing technology (Quail et al. 2012) which cannot be overcome with short $k$-mers. 74). In the two false negative cases, both contained all 7 typing genes, with one containing 2 copies of gene phoE which Krocus was unable to distinguish, and one containing a variant in fum $C$ which was not in the typing database.

202

\section{ONT results}

204 For all 12 K. pneumoniae samples (100\%) of uncorrected ONT reads Krocus provided the 205 expected sequence type. The mean time to the expected sequence type was 134 seconds after 206 a mean of 3250 reads. As a comparison, de novo assembled genomes using the ONT reads 207 alone from (R. R. Wick et al. 2017) did not identify any of the sequence types when analysed by 208 TS-mlst. This was due to the inherent high base error rate which resulted in a poor quality 209 assembly. Only hybrid assemblies additionally utilising Illumina short read data could be 210 sequence typed. This gives Krocus an advantage over de novo assembly of ONT only reads.

211 For the simulated E.coli reference genome reads, 2 flow cell models were used, R9 1D and R9 212 2D. The lower quality R9 1D simulations correctly identified the STs in $432(82.4 \%)$ cases with a mean running time of 71 seconds and a mean of 6618 reads. Of the 92 STs which were not called correctly, 84 were as a result of errors in calling fumC or gyrB. The higher quality R9 2D simulations correctly identified STs in 492 (93.8\%) cases with a mean running time of 25 seconds and after a mean of 5894 reads. Of the 36 STs which were not called correctly, 34 were

217 as a result of errors in calling fumC or gyrB. The difference in running time is due to lower error

218 rates in the $\mathrm{R} 92 \mathrm{D}$ uncorrected reads, reducing the number of observed $k$-mers. The quality of 
219 the base called data used for generating the simulation models directly impacts the ability to call 220 STs accurately.

\section{Discussion}

222

223

224

225

226

227

228

229

230

231

232

233

234

235

236

237

238

239

240

241

242

As shown previously it is possible to call sequence types directly from uncorrected long read data. Whilst the error rates in uncorrected reads are high, the error profile of long reads is such that short regions of high quality data exist between the errors in the reads. Read correction typically overlaps reads and calls a consensus to fix errors in the underlying read. The ability to utilise uncorrected reads and still generate accurate results means that time consuming read correction steps are not needed to generate sequence typing information. Krocus achieves this by using short $k$-mers.

The PacBio sequencer produces uncorrected reads in both BAM format and HDF5 format (legacy). These are not available to call in real-time due to the requirement for post-sequencing base calling, so Krocus would only ever be run on the final data at the end of a sequencing run. The ONT sequencers can produce uncorrected reads in FASTQ format in real-time. These can be streamed directly into Krocus taking maximum advantage of the method. Additionally, as the ONT sequencers can be halted mid-run, flushed and loaded with a new sample, a single flow cell can be reused many times over, potentially as soon as Krocus has generated a sequence type. This has the effect of reducing the costs of the sequencing run.

Due to a current lack of large ONT datasets in the public archives, simulated data was used. Simulated ONT reads gives an insight into the performance of Krocus with different error models, with more accurate read sets resulting in more accurate ST calling. The ONT sequencing technology and the base calling software are currently undergoing rapid change with constant improvements in the quality of the data, so these simulations should seen as a baseline performance for Krocus.

\section{Conclusions}

Krocus is the only tool which can call sequence types directly from uncorrected long reads with high accuracy. The sensitivity of $94 \%$ and specificity of $97 \%$ achieved by Krocus on a large, diverse, PacBio dataset is similar to gold standard experimental standard methods (Liu et al. 2012). By calling sequence types directly from uncorrected long reads, the need for post processing steps and de novo assembly is eliminated, reducing the turnaround time for MLST from days to minutes. For a small ONT dataset of real samples, Krocus correctly called the sequence type in all cases, compared to de novo assemblies of the same data, where no sequence types could be called. In a large simulated ONT dataset of E. coli, the sensitivity was $82-94 \%$, depending on the flowcell modelled.

\section{Acknowledgements}

We wish to thank Nick Grayson from the Wellcome Sanger Institute for assistance with the NCTC 3000 dataset. Thanks to João Carriço and Nabil-Fareed Alikhan for providing helpful feedback and suggestions for this paper. Thank you to Karel Brrinda and Nick Loman for reviewing this paper and providing helpful feedback. 


\section{References}

259

260

261

262

263

264

265

266

267

268

269

270

271

272

273

274

275

276

277

278

279

280

281

282

283

284

285

286

287

288

289

290

291

292

293

294

295

296

297

298

299

300

301

302

303

304

305

306
Afgan E, Sloggett C, Goonasekera N, Makunin I, Benson D, Crowe M, Gladman S, Kowsar Y, Pheasant M, Horst R, Lonie A., Genomics Virtual Laboratory: A Practical Bioinformatics Workbench for the Cloud., PLoS One. 2015 Oct 26;10(10):e0140829.

Connor T, Loman N, Thompson S, Smith A, Southgate J, Poplawski R, Bull M, Richardson E, Ismail M, Thompson S, Kitchen C, Guest M, Bakke M, Sheppard S, Pallen M. 01/09/2016. M Gen 2(9): doi:10.1099/mgen.0.000086

Chin, Chen-Shan, David H. Alexander, Patrick Marks, Aaron A. Klammer, James Drake, Cheryl Heiner, Alicia Clum, Alex Copeland, John Huddleston, Evan E Eichler, Stephen W Turner, and Jonas Korlach. 2013. "Nonhybrid, Finished Microbial Genome Assemblies from LongRead SMRT Sequencing Data." Nature Methods 10 (6):563-69.

Faria, N. R., J. Quick, I. M. Claro, J. Thézé, J. G. de Jesus, M. Giovanetti, M. U. G. Kraemer, S. C. Hill, A. Black, A. C. da Costa, L. C. Franco, S. P. Silva, C.-H. Wu, J. Raghwani, S. Cauchemez, L. du Plessis, M. P. Verotti, W. K. de Oliveira, E. H. Carmo, G. E. Coelho, A. C. F. S. Santelli, L. C. Vinhal, C. M. Henriques, J. T. Simpson, M. Loose, K. G. Andersen, N. D. Grubaugh, S. Somasekar, C. Y. Chiu, J. E. Muñoz-Medina, C. R. Gonzalez-Bonilla, C. F. Arias, L. L. Lewis-Ximenez, S. A. Baylis, A. O. Chieppe, S. F. Aguiar, C. A. Fernandes, P. S. Lemos, B. L. S. Nascimento, H. A. O. Monteiro, I. C. Siqueira, M. G. de Queiroz, T. R. de Souza, J. F. Bezerra, M. R. Lemos, G. F. Pereira, D. Loudal, L. C. Moura, R. Dhalia, R. F. França, T. Magalhães, E. T. Marques Jr, T. Jaenisch, G. L. Wallau, M. C. de Lima, V. Nascimento, E. M. de Cerqueira, M. M. de Lima, D. L. Mascarenhas, J. P. Moura Neto, A. S. Levin, T. R. Tozetto-Mendoza, S. N. Fonseca, M. C. Mendes-Correa, F. P. Milagres, A. Segurado, E. C. Holmes, A. Rambaut, T. Bedford, M. R. T. Nunes, E. C. Sabino, L. C. J. Alcantara, N. J. Loman and O. G. Pybus. 2017. "Establishment and Cryptic Transmission of Zika Virus in Brazil and the Americas." Nature 546 (7658):406-10.

Feijao, Pedro, Hua-Ting Yao, Dan Fornika, Jennifer Gardy, William Hsiao, Cedric Chauve, and Leonid Chindelevitch. 2018. "MentaLiST - A Fast MLST Caller for Large MLST Schemes." Microbial Genomics. https://doi.org/10.1099/mgen.0.000146.

Gardy, Jennifer L., and Nicholas J. Loman. 2018. "Towards a Genomics-Informed, Real-Time, Global Pathogen Surveillance System." Nature Reviews. Genetics 19 (1):9-20.

Gupta, Anuj, I. King Jordan, and Lavanya Rishishwar. 2017. "stringMLST: A Fast K-Mer Based Tool for Multilocus Sequence Typing." Bioinformatics 33 (1):119-21.

Hunt, Martin, Nishadi De Silva, Thomas D. Otto, Julian Parkhill, Jacqueline A. Keane, and Simon R. Harris. 2015. "Circlator: Automated Circularization of Genome Assemblies Using Long Sequencing Reads." Genome Biology 16 (December):294.

Jolley, Keith A., and Martin C. J. Maiden. 2010. "BIGSdb: Scalable Analysis of Bacterial Genome Variation at the Population Level." BMC Bioinformatics 11 (December):595.

Koren, Sergey, Brian P. Walenz, Konstantin Berlin, Jason R. Miller, Nicholas H. Bergman, and Adam M. Phillippy. 2017. "Canu: Scalable and Accurate Long-Read Assembly via Adaptive K-Mer Weighting and Repeat Separation." Genome Research 27 (5):722-36.

Li, Heng. 2016. "Minimap and Miniasm: Fast Mapping and de Novo Assembly for Noisy Long Sequences." Bioinformatics 32 (14): 2103-10.

Liu, Lin, Yinhu Li, Siliang Li, Ni Hu, Yimin He, Ray Pong, Danni Lin, Lihua Lu, and Maggie Law. 2012. "Comparison of Next-Generation Sequencing Systems." BioMed Research International 2012 (July). Hindawi. https://doi.org/10.1155/2012/251364.

Page, Andrew J., Nabil-Fareed Alikhan, Heather A. Carleton, Torsten Seemann, Jacqueline A. Keane, and Lee S. Katz. 2017. "Comparison of Classical Multi-Locus Sequence Typing Software for next-Generation Sequencing Data." Microbial Genomics 3 (8):e000124.

Quail, Michael A., Miriam Smith, Paul Coupland, Thomas D. Otto, Simon R. Harris, Thomas R. 
Connor, Anna Bertoni, Harold P. Swerdlow, and Yong Gu. 2012. "A Tale of Three next Generation Sequencing Platforms: Comparison of Ion Torrent, Pacific Biosciences and Illumina MiSeq Sequencers." BMC Genomics 13 (July):341.

Quick, Joshua, Philip Ashton, Szymon Calus, Carole Chatt, Savita Gossain, Jeremy Hawker, Satheesh Nair, Keith Neal, Kathy Nye, Tansy Peters, Elizabeth De Pinna, Esther Robinson, Keith Struthers, Mark Webber, Andrew Catto, Timothy J. Dallman, Peter Hawkey, Nicholas J. Loman. 2015. "Rapid Draft Sequencing and Real-Time Nanopore Sequencing in a Hospital Outbreak of Salmonella." Genome Biology 16 (1). https://doi.org/10.1186/s13059$\underline{015-0677-2 .}$

Vaser, Robert, Ivan Sović, Niranjan Nagarajan, and Mile Šikić. 2017. "Fast and Accurate de Novo Genome Assembly from Long Uncorrected Reads." Genome Research 27 (5): 73746.

Votintseva, Antonina A., Phelim Bradley, Louise Pankhurst, Carlos Del Ojo Elias, Matthew Loose, Kayzad Nilgiriwala, Anirvan Chatterjee, E. Grace Smith, Nicolas Sanderson, Timothy M. Walker, Marcus R. Morgan, David H. Wyllie, A. Sarah Walker, Tim E. A. Peto, Derrick W. Crook, Zamin Iqbal. 2017. "Same-Day Diagnostic and Surveillance Data for Tuberculosis via Whole-Genome Sequencing of Direct Respiratory Samples." Journal of Clinical Microbiology 55 (5):1285-98.

Wick, Ryan. 2017a. "Basecalled ONT Reads," July. https://doi.org/10.4225/49/595c46ae5efb4. . 2017b. "Unicycler (v0.4.0) + Nanopolish (v0.7.0) Assemblies (ONT-Only)," July. https://doi.org/10.4225/49/595c4b31defd0.

Wick, Ryan R., Louise M. Judd, Claire L. Gorrie, and Kathryn E. Holt. 2016. "Unicycler: Resolving Bacterial Genome Assemblies from Short and Long Sequencing Reads." https://doi.org/10.1101/096412.

Wick, Ryan, Louise M. Judd, and Kathryn E. Holt. 2018. "Comparison of Oxford Nanopore Basecalling Tools," January. https://doi.org/10.5281/zenodo.1134473.

Wick, Ryan R., Louise M. Judd, Claire L. Gorrie, and Kathryn E. Holt. 2017. "Completing Bacterial Genome Assemblies with Multiplex MinION Sequencing." Microbial Genomics 3 (10):e000132.

Yang C, Chu J, Warren RL, Birol I. 2017. "NanoSim: nanopore sequence read simulator based on statistical characterization." Gigascience. 2017 Apr 1;6(4):1-6. doi:

10.1093/gigascience/gix010. 


\section{Figure 1 (on next page)}

Flowchart of the Krocus method.

The square boxes denote processes that the data undergoes, the diamond shapes denote a decision point, the box with a wavy lower line denotes a data file, and the tabs denote an output. 


\section{Figure 2 (on next page)}

The reads and time to correctly predict an ST for each PacBio NCTC species analysed.

(A) Number of reads analysed before the Krocus correctly predicted an ST for each PacBio NCTC species analysed. (B) Time in seconds before Krocus correctly predicted an ST for each PacBio NCTC species analysed. 
A

150000 -

\section{$\mathrm{B}^{\text {Manuscript to be reviewed }}$}

600 -

100000 -

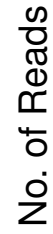

50000 -

0 -

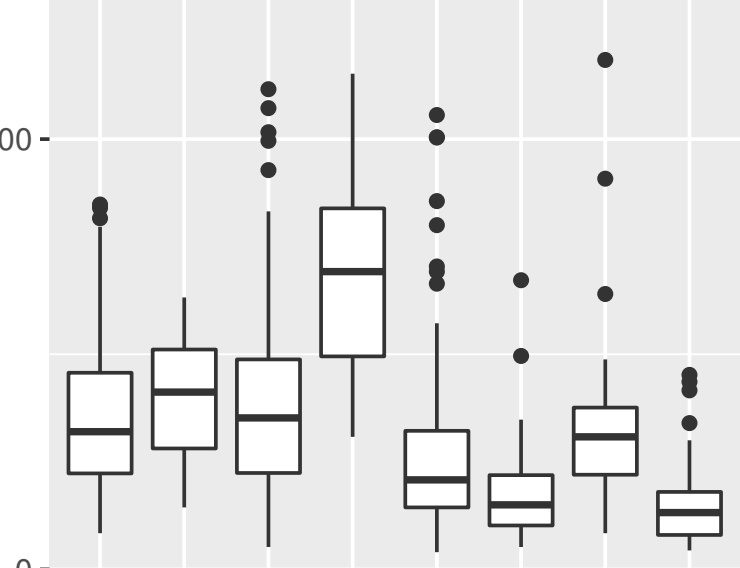

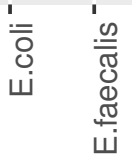

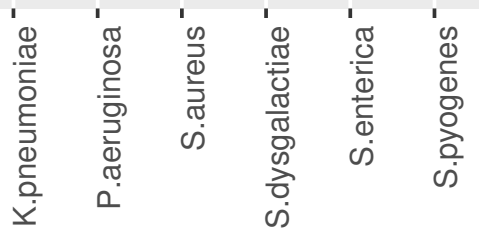

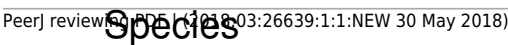

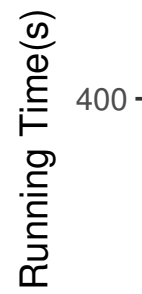

200 -

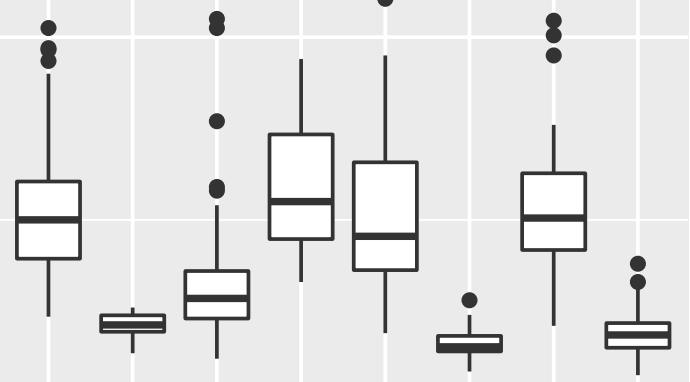

0 -

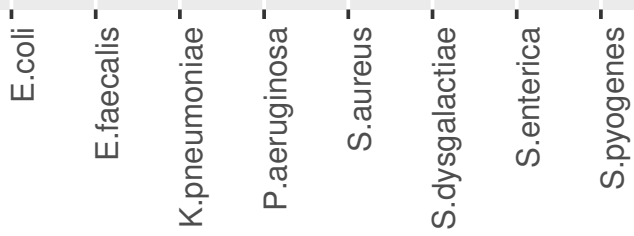
Species 


\section{Table $\mathbf{1}$ (on next page)}

NCTC 3000 PacBio sequenced samples with results after analysis with Krocus.

An ST is said to be in agreement if it matches the ST called by TS-mlst from a de novo assembly. 


\begin{tabular}{|c|c|c|c|c|c|}
\hline Species & $\begin{array}{l}\text { No. } \\
\text { Samples }\end{array}$ & $\begin{array}{l}\text { No. of } \\
\text { unique STs }\end{array}$ & $\begin{array}{l}\text { No. in } \\
\text { agreement }\end{array}$ & $\begin{array}{l}\text { Mean wall } \\
\text { time }(s)\end{array}$ & Mean Reads \\
\hline E. coli & 226 & 204 & 204 & 102 & 17524 \\
\hline E. faecalis & 11 & 10 & 10 & 42 & 19900 \\
\hline K. pneumoniae & 113 & 101 & 109 & 62 & 22297 \\
\hline$P$. aeruginosa & 22 & 21 & 18 & 127 & 41444 \\
\hline S. aureus & 114 & 92 & 111 & 122 & 16255 \\
\hline S. dysgalactiae & 16 & 16 & 16 & 32 & 10412 \\
\hline S. enterica & 48 & 46 & 47 & 107 & 16348 \\
\hline S. pyogenes & 47 & 47 & 47 & 37 & 7714 \\
\hline Other & 112 & 101 & 106 & 57 & 15024 \\
\hline Total & 709 & 638 & 668 & 86 & 17439 \\
\hline
\end{tabular}

